\title{
The distribution of atomic gas and dust in nearby galaxies - II. Further matched-resolution Very Large Array H I and SCUBA 850- $\mu \mathrm{m}$ images
}

\author{
H. C. Thomas, ${ }^{1 \star}$ L. Dunne, ${ }^{2}$ D. A. Green, ${ }^{1} \dagger$ M. S. Clemens,${ }^{3}$ P. Alexander ${ }^{1}$ \\ and S. Eales ${ }^{2}$ \\ ${ }^{1}$ Mullard Radio Astronomy Observatory, Cavendish Laboratory, Madingley Road, Cambridge CB3 OHE \\ ${ }^{2}$ Department of Physics and Astronomy, University of Wales Cardiff, PO Box 913, Cardiff CF2 $3 Y B$ \\ ${ }^{3}$ Dipartimento di Astronomia, Universita degli Studi di Padova, Vicolo dell'Osservatorio, 2, 35122 Padova, Italy
}

Accepted 2003 November 16. Received 2003 November 10; in original form 2003 October 3

\begin{abstract}
We present Very Large Array (VLA) C-array 21-cm H I images of galaxies from the SCUBA Local Universe Galaxy Survey which have been observed at $850 \mu \mathrm{m}$ with the James Clerk Maxwell Telescope. Matched-resolution $(\sim 25 \mathrm{arcsec}) \mathrm{H}$ I images of 17 galaxies are presented and compared with $850-\mu \mathrm{m}$ images. $\mathrm{H}$ I or $850-\mu \mathrm{m}$ images of an additional six galaxies which were detected at only one wavelength are presented. Additionally, lower resolution $\mathrm{H}$ i observations of nine galaxies are presented. The observations of these galaxies, along with results previously presented, do not show any obvious trends in the $\mathrm{H}_{\mathrm{I}}$ /dust or $\mathrm{H}_{2}$ /dust mass ratios with morphological type.
\end{abstract}

Key words: galaxies: abundances - galaxies: ISM - galaxies: structure - radio lines: galaxies.

\section{INTRODUCTION}

The Submillimetre Common-User Bolometer Array (SCUBA) Local Universe Galaxy Survey (SLUGS, see Dunne et al. 2000; Dunne \& Eales 2001; Thomas et al. 2002) is a major survey of dust emission in 104 nearby galaxies, as observed with the SCUBA instrument on the James Clerk Maxwell Telescope (JCMT). Although it provides important data on the dust emission from a much larger number of galaxies than has previously been available, comparison with observations of other components of the ISM of the galaxies is also valuable. This is the second of a series of three papers in which we investigate the relationship of the dust emission to the neutral hydrogen (H I) emission. In (Thomas et al. (2002, hereafter Paper I), matched-resolution H I images of 20 SLUGS galaxies from Very Large Array (VLA) C-array observations were presented and compared with the 850- $\mu \mathrm{m}$ images. Here similar data for a further 24 SLUGS galaxies are presented, together with lower resolution VLA D-array H I observations of some larger ( $>2.5 \mathrm{arcmin}$ ) galaxies from the SLUGS sample. The observations and the data reduction are briefly discussed in Section 2, and the results presented in Section 3. The overall properties of the dust and $\mathrm{H}_{\mathrm{I}}$ in the galaxies presented here and in Paper I are discussed in Section 4. Analysis and discussion of their radial distributions are the subjects of a future paper (Thomas et al. 2004, Paper III). The lower resolution D-array observations are discussed in the Appendix.

\footnotetext{
${ }^{\star}$ Present address: 12 Middlehurst Ave, Weaverham, Northwich CW8 3BW.
} †E-mail: dag@mrao.cam.ac.uk

\section{VLA OBSERVATIONS AND DATA REDUCTION}

Galaxies were selected from the SLUGS sample with angular sizes $\left(D_{25}\right)$ less than 3 arcmin. From this we chose 24 galaxies to observe at $21 \mathrm{~cm}$ with the VLA in C-array in 2001 July. Table 1 lists the sample. These 24 galaxies consist of 13 IRAS selected galaxies, plus 11 optically selected (see Dunne et al. 2000; Dunne 2000, and Table 1), whereas the galaxies in Paper I consisted solely of IRAS selected galaxies. The observations were made, and the data reduced, in a similar way to that described in detail in Paper I (see Section 3.1). In addition, a small sample of 13 SLUGS galaxies larger than 2.5 arcmin in size were observed in 1999 April with the VLA in D-array. These observations are of lower resolution (typically 50 arcsec) than the SCUBA $850-\mu \mathrm{m}$ observations, and therefore do not provide matched-resolution $\mathrm{H}$ I images. However, the D-array observations are more sensitive to emissions on larger scales, and are useful to determine the overall extent of $\mathrm{HI}$ in larger galaxies. Three of the galaxies (NGC 958, 5020 and 5962) observed in D-array were also observed in C-array. Of the 10 other galaxies observed in D-array, nine were detected, and the results for these galaxies are presented in the Appendix.

\section{RESULTS}

Of the 24 galaxies observed in $\mathrm{HI}$, emissions from 17 were clearly detected and one was barely detected (NGC 7047, see discussion below). Five galaxies were not detected in H I (IC 5090, IC 1368 and NGC 6120, detected at $850 \mu \mathrm{m}$; IC 1211 and UGC 10500, not detected at $850 \mu \mathrm{m}$ ), and one (Arp 220) was detected in absorption. 
Table 1. General properties of the sample.

\begin{tabular}{|c|c|c|c|c|c|c|c|}
\hline (1) & $\begin{array}{c}\text { RA } \\
(\mathrm{B} 1950) \\
(2)\end{array}$ & $\begin{array}{c}\text { Dec. } \\
\text { (B1950) } \\
(3)\end{array}$ & Type & $\begin{array}{c}\text { Nuclear type } \\
\text { (5) }\end{array}$ & $\begin{array}{c}\text { Distance } \\
75 / H_{0}(\mathrm{Mpc}) \\
(6)\end{array}$ & $\begin{array}{c}\log _{10}\left(L_{\mathrm{FIR}}\right) \\
\left(\mathrm{L}_{\odot}\right) \\
(7)\end{array}$ & Sample \\
\hline UGC 148 & 001316.3 & +154843 & S? & - & 57.1 & 10.34 & $\mathrm{O}$ \\
\hline NGC 99 & 002123.8 & +152937 & Scd & - & 70.1 & 10.14 & $\mathrm{O}$ \\
\hline NGC 958 & 022811.0 & -030944 & $\mathrm{Sc}$ & - & 79.9 & 10.97 & I \\
\hline NGC 992 & 023435.6 & +205304 & S? & - & 54.2 & 10.78 & I \\
\hline NGC 1222 & 030625.6 & -030843 & E/S0 & - & 33.6 & 10.36 & I \\
\hline UGC 2982 & 040943.0 & +052512 & $\mathrm{Sm}$ & $\mathrm{H}_{\text {II }}$ & 67.0 & 10.99 & I \\
\hline NGC 4433 & 122503.8 & -080009 & $\mathrm{Sab}$ & - & 41.9 & 10.64 & I \\
\hline NGC 4793 & 125215.6 & +291234 & $\mathrm{Sc}$ & - & 36.7 & 10.54 & I \\
\hline NGC 5020 & 131010.7 & +125153 & $\mathrm{Sbc}$ & - & 44.8 & 10.38 & I \\
\hline NGC 5937 & 152809.9 & -023933 & $\mathrm{Sb}$ & - & 33.9 & 10.83 & I \\
\hline NGC 5953/4 & 153213.2 & +152137 & $\mathrm{Sa} / \mathrm{Scd}$ & Sy2/Sy2 & 22.3 & 10.21 & I \\
\hline Arp 220 & 153246.8 & +234008 & S? & H II; Sy2 & 75.8 & 11.94 & I \\
\hline NGC 5962 & 153414.0 & +164621 & $\mathrm{Sc}$ & $\mathrm{H}_{\text {II }}$ & 29.6 & 10.19 & I \\
\hline UGC 10200 & 160403.7 & +412843 & $\mathrm{~S} ?$ & $\mathrm{H}_{\text {II }}$ & 25.7 & - & $\mathrm{O}$ \\
\hline IC 1211 & 161538.2 & +530740 & $\mathrm{E}$ & - & 75.4 & - & $\mathrm{O}$ \\
\hline NGC 6120 & 161801.2 & +375336 & $\mathrm{~S} ?$ & $\mathrm{H}_{\mathrm{II}}$ & 121.9 & 11.13 & $\mathrm{O}$ \\
\hline UGC 10500 & 163805.1 & +574917 & $\mathrm{~S} 0 / \mathrm{a}$ & - & 71.9 & - & $\mathrm{O}$ \\
\hline IC 5090 & 210855.0 & -021417 & $\mathrm{Sa}$ & - & 120.0 & 11.14 & $\mathrm{O}$ \\
\hline IC 1368 & 211140.4 & +015813 & $\mathrm{Sa}$ & Sy 2 & 52.4 & 10.34 & $\mathrm{O}$ \\
\hline NGC 7047 & 211353.0 & -010208 & $\mathrm{Sb}$ & - & 75.9 & 10.05 & $\mathrm{O}$ \\
\hline NGC 7081 & 212852.1 & +021612 & $\mathrm{Sb}$ & - & 44.3 & 9.93 & $\mathrm{O}$ \\
\hline NGC 7591 & 231543.9 & +061845 & $\mathrm{Sbc}$ & LINER & 63.8 & 10.84 & I \\
\hline NGC 7722 & 233609.3 & +154039 & $\mathrm{~S} 0 / \mathrm{a}$ & - & 50.6 & 10.05 & $\mathrm{O}$ \\
\hline UGC $12914 / 5$ & 235906.8 & +231255 & $\mathrm{Scd} / \mathrm{Sc}$ & LINER/LINER & 63.0 & 10.70 & I \\
\hline
\end{tabular}

Notes: (1) Galaxy name. (2) and (3) RA and Dec. (B1950). (4) Morphological type. (5) Spectral type. (6) Distance, from recession velocity. (7) For IRAS-selected SLUGS galaxies, far-infrared luminosity from Dunne et al. (2000) and Dunne (2000). (8) Sample, either 'I' for infrared or ' $\mathrm{O}$ ' for optically selected.

The total H I flux density of each galaxy, or a limit, was determined from the integrated $\mathrm{H}$ I images. For galaxies not detected, $3 \sigma_{\text {rms }}$ upper limits on the amount of $\mathrm{HI}$ were estimated by assuming a typical velocity width of $350 \mathrm{~km} \mathrm{~s}^{-1}$. These results are given in Table 2. As found in Paper I, the VLA observations recover typically $\sim 70$ per cent of the total $\mathrm{H}$ I flux found in single-dish $\mathrm{H}$ I observations in the literature. The missing flux is due to the limited sensitivity of the C-array observations to extended emission on scales greater than about 3 arcmin, i.e. on scales larger than those measured by the SCUBA $850-\mu \mathrm{m}$ observations.

The matched-resolution ( 25 arcsec) $\mathrm{H} \mathrm{I}$ and $850-\mu \mathrm{m}$ images of 17 of the selected SLUGS galaxies detected in both $\mathrm{HI}$ and at $850 \mu \mathrm{m}$ are shown in Fig. 1, where the field-of-view has been chosen to match the extent of the 850- $\mu$ m data. In four of these galaxies (NGC 4793, 5020, 5962 and 7591), the extent of the $\mathrm{HI}$ emission is much larger than that of the SCUBA field of view - the H I images from these galaxies over wider fields of view are shown in Fig. 2. A wide-field view of the H I detected in UGC 10200, which was not detected at $850 \mu \mathrm{m}$, is shown in Fig. 3. SCUBA 850- $\mu \mathrm{m}$ images of the galaxies not detected (NGC 6120, NGC 5090 and IC 1368), or only weakly detected in H I (NGC 7047) are shown in Fig. 4.

\subsection{Notes on some individual sources}

\subsubsection{NGC 958}

At $850 \mu \mathrm{m}$ the small companion galaxy to the south-east was also detected, although the emission does not appear to be well correlated with the HI. The H I distribution is somewhat irregular beyond the edge of the optical disc of NGC 958 and extends towards the south.
The lower-resolution, larger field D-array H I image of NGC 958, shown in Fig. 5, reveals a faint bridge of emissions stretching to a small companion galaxy to the north-west.

\subsubsection{NGC 992}

The H I distribution of NGC 992 shows diffuse emissions extending to Mrk 369, a small elliptical 2.6 arcmin to the north-west. The mean recession velocity of Mrk 369, as determined from the $\mathrm{HI}$ data, is $4050 \mathrm{~km} \mathrm{~s}^{-1}$ which is consistent with the optical velocity of $4045 \mathrm{~km}$ $\mathrm{s}^{-1}$ (Huchra, Vogeley \& Geller 1999). There is likely to be diffuse, extended $\mathrm{H}$ I associated with the pair which was not detected by this snap-shot observation, as single-dish observations detect more $\mathrm{H}$ I flux.

\subsubsection{NGC 5937}

The $850-\mu \mathrm{m}$ emission is concentrated towards the optical centre of this galaxy, whereas the HI associated with NGC 5937 is significantly offset from its centre. NGC 5937 has no nearby companions.

\subsubsection{Arp 220}

This is the most luminous infrared galaxy in the IRAS bright galaxy sample, the brightest at $850 \mu \mathrm{m}$. It is at a distance of $72.5 \mathrm{Mpc}$, and has a far-infrared luminosity of $\sim 10^{12} \mathrm{~L} \odot$. It is the closest, and hence most well studied, of all ultraluminous infrared galaxies (e.g. Soifer et al. 1999; Anantharamaiah et al. 2000; Wiedner 
Table 2. Gas and dust masses.

\begin{tabular}{|c|c|c|c|c|c|c|c|}
\hline (1) & $\begin{array}{c}S_{\mathrm{H} \mathrm{I}} \\
\left(\mathrm{Jy} \mathrm{km} \mathrm{s}^{-1}\right) \\
(2)\end{array}$ & $\begin{array}{c}\log _{10}\left(M_{\mathrm{H}_{\mathrm{I}}}\right) \\
\left(\mathrm{M}_{\odot}\right) \\
(3)\end{array}$ & $\begin{array}{c}\log _{10}\left(M_{\mathrm{d}}\right) \\
\left(\mathrm{M}_{\odot}\right) \\
(4)\end{array}$ & $\begin{array}{c}\log _{10}\left(M_{\mathrm{H}_{2}}\right) \\
\left(\mathrm{M}_{\odot}\right) \\
(5)\end{array}$ & $\begin{array}{l}\text { Refs } \\
\text { (6) }\end{array}$ & $\begin{array}{c}M_{\mathrm{H}_{2}} / M_{\mathrm{HI}} \\
(7)\end{array}$ & $\begin{array}{c}\left(M_{\mathrm{H} 2}+M_{\mathrm{H} I}\right) / M_{\mathrm{d}} \\
(8)\end{array}$ \\
\hline UGC 148 & $6.1 \pm 0.5$ & 9.67 & 7.76 & - & & - & - \\
\hline NGC 99 & $12.6 \pm 1.3$ & 10.16 & 7.77 & - & & - & - \\
\hline NGC 958 & $26.0 \pm 2.7$ & 10.59 & 8.34 & 10.21 & $a, b$ & 0.42 & 252 \\
\hline NGC 992 & $9.0 \pm 1.1$ & 9.79 & 7.74 & 9.77 & $\mathrm{a}, \mathrm{c}, \mathrm{d}, \mathrm{e}, \mathrm{f}$ & 0.95 & 219 \\
\hline NGC 1222 & $6.9 \pm 0.8$ & 9.26 & 7.07 & - & & - & - \\
\hline UGC 2982 & $5.8 \pm 0.6$ & 9.79 & 7.99 & 10.06 & $c, g$ & 1.86 & 180 \\
\hline NGC 4433 & $12.4 \pm 1.3$ & 9.71 & 7.70 & 9.62 & $\mathrm{~g}$ & 0.81 & 186 \\
\hline NGC 4793 & $32.5 \pm 3.6$ & 10.01 & 7.64 & 9.49 & $\mathrm{a}, \mathrm{g}, \mathrm{h}$ & 0.30 & 305 \\
\hline NGC 5020 & $23.0 \pm 2.4$ & 10.04 & 7.73 & 9.30 & $\mathrm{i}$ & 0.18 & 241 \\
\hline NGC 5937 & $2.2 \pm 0.5$ & 8.78 & 7.57 & - & & - & - \\
\hline NGC 5953/4 & $6.1 \pm 0.6$ & 8.86 & 7.30 & 8.99 & $\mathrm{j}, \mathrm{k}$ & 1.35 & 85 \\
\hline Arp 220 & A & $<10.64$ & 8.79 & 10.44 & $a, b, g$ & $>0.63$ & - \\
\hline NGC 5962 & $18.4 \pm 1.9$ & 9.58 & 7.56 & 9.67 & $\mathrm{~g}, \mathrm{k}$ & 1.23 & 233 \\
\hline UGC 10200 & $8.5 \pm 1.0$ & 9.12 & $<6.17$ & - & & - & - \\
\hline IC 1211 & $<0.7$ & $<8.97$ & $<7.13$ & - & & - & - \\
\hline NGC 6120 & $<1.7$ & $<9.78$ & 8.09 & - & & - & - \\
\hline UGC 10500 & $<2.1$ & $<9.41$ & - & - & & - & - \\
\hline IC 5090 & $<1.9$ & $<9.81$ & 8.36 & - & & - & - \\
\hline IC 1368 & $<2.0$ & $<9.11$ & 7.17 & - & & - & - \\
\hline NGC 7047 & $<1.8$ & $<9.39$ & 7.60 & - & & - & - \\
\hline NGC 7081 & $7.3 \pm 0.8$ & 9.53 & 7.16 & - & & - & - \\
\hline NGC 7591 & $22.5 \pm 2.4$ & 10.33 & 7.85 & 10.23 & $\mathrm{f}$ & 0.79 & 541 \\
\hline NGC 7722 & $1.4 \pm 0.3$ & 8.93 & 7.52 & 9.62 & 1 & 4.90 & 152 \\
\hline UGC $12914 / 5$ & $9.6 \pm 1.0$ & 9.95 & 8.00 & 10.41 & $\mathrm{~m}$ & 2.88 & 346 \\
\hline
\end{tabular}

Notes: (1) Galaxy name. (2) VLA H I flux and error. 'A' indicates the galaxy was only observed in absorption. (3) The H I gas mass, calculated from $2.36 \times 10^{5} D^{2} S_{\mathrm{H}_{\mathrm{I}}}$, where $D$ is the distance to the source in Mpc. (4) Dust mass taken from Dunne et al. (2000). (5) Molecular gas mass, assuming a CO-to- $\mathrm{H}_{2}$ conversion factor of $X=2.8 \times 10^{20} \mathrm{H}_{2} \mathrm{~cm}^{-2}$ (Kenney \& Young 1989). (6) References for the CO fluxes are: (a) Sanders, Scoville \& Soifer (1991); (b) Maiolino et al. (1997); (c) Chini, Krügel \& Lemke (1996); (d) Elfhag et al. (1996); (e) Sanders \& Mirabel (1985); (f) Lavezzi \& Dickey (1998); (g) Young et al. (1995); (h) Casoli et al. (1996); (i) Seaquist, private communication; (j) Sofue et al. (1993); (k) Tinney et al. (1990); (1) Wang et al. (1992); (m) Zhu et al. (1999). (7) Molecular gas to atomic gas mass ratio [calculated from columns (3) and (5)]. (8) Gas-to-dust mass ratio [calculated from columns (3) to (5)].

et al. 2002; McDowell et al. 2003). Arp 220 showed strong H I absorption.

\subsubsection{NGC 7047}

This galaxy was a weak detection at $850 \mu \mathrm{m}$. The Hi line profile reveals only weak $(\sim 2 \sigma)$ emission around the systemic velocity of $5650 \mathrm{~km} \mathrm{~s}^{-1}$ when integrated over the galaxy. Theureau et al. (1998) recorded an $\mathrm{HI}$ flux of $0.9 \mathrm{Jy} \mathrm{km} \mathrm{s}^{-1}$ which is consistent with the upper limit derived from these observations.

\subsubsection{NGC 7722}

Although the H I emission associated with NGC 722 is weak, it is aligned along the dust lane visible in the optical image. The dip in the HI approximately coincides with the peak in the $850-\mu \mathrm{m}$ emission. Although there is a radio continuum source at the centre of the galaxy, it is too weak to explain the dip in the $\mathrm{HI}$ as the result of absorption. Wang, Kenney \& Ishizuki (1992) observed NGC 7722 in CO $(J=1 \rightarrow 0$, with a beam of 16 arcsec $)$ and found a molecular gas mass of $\sim 10^{9} \mathrm{M}_{\odot}$. Although this observation was positioned at the radio centre (rather than at the H I dip), this galaxy is rich in molecular gas which may explain the lack of $\mathrm{HI}$.

\subsubsection{UGC 12914/5}

The Hi image of UGC 12914/5 (the 'Taffy' galaxies) is in good agreement with the C-array VLA image of Condon et al. (1993, their fig. 4). However, less $\mathrm{H}$ I flux (9.6 Jy km s${ }^{-1}$ compared with $15.3 \mathrm{Jy} \mathrm{km} \mathrm{s}^{-1}$ ) is recovered from the present observations, due to the fact that Condon et al. performed a full synthesis $(10 \mathrm{~h})$ observation, and they were therefore more sensitive to emission on larger angular scales. Radio continuum observations by Condon et al. revealed that approximately half of the 1.4-GHz emission arises between the two galaxies. This excess emission gives UGC 12914/5 the lowest far-infrared/radio ratio of all the 258 galaxies in the IRAS bright galaxy survey. Condon et al. propose a bridge of cosmic rays, magnetic fields and $\mathrm{H}$ I gas have been stripped from the discs of the galaxies, after a head-on collision $\sim 2 \times 10^{7} \mathrm{yr}$ ago. The $850-\mu \mathrm{m}$ image shows tentative evidence for a bridge of $850-\mu \mathrm{m}$ emission between these two galaxies, which approximately follows the peak H I contour.

\section{DISCUSSION}

\subsection{Gas and dust morphology}

As was seen in Paper I, it is rare for the $850-\mu \mathrm{m}$ and H I peaks to coincide. In many cases the $850-\mu \mathrm{m}$ peak is found at the location of a 

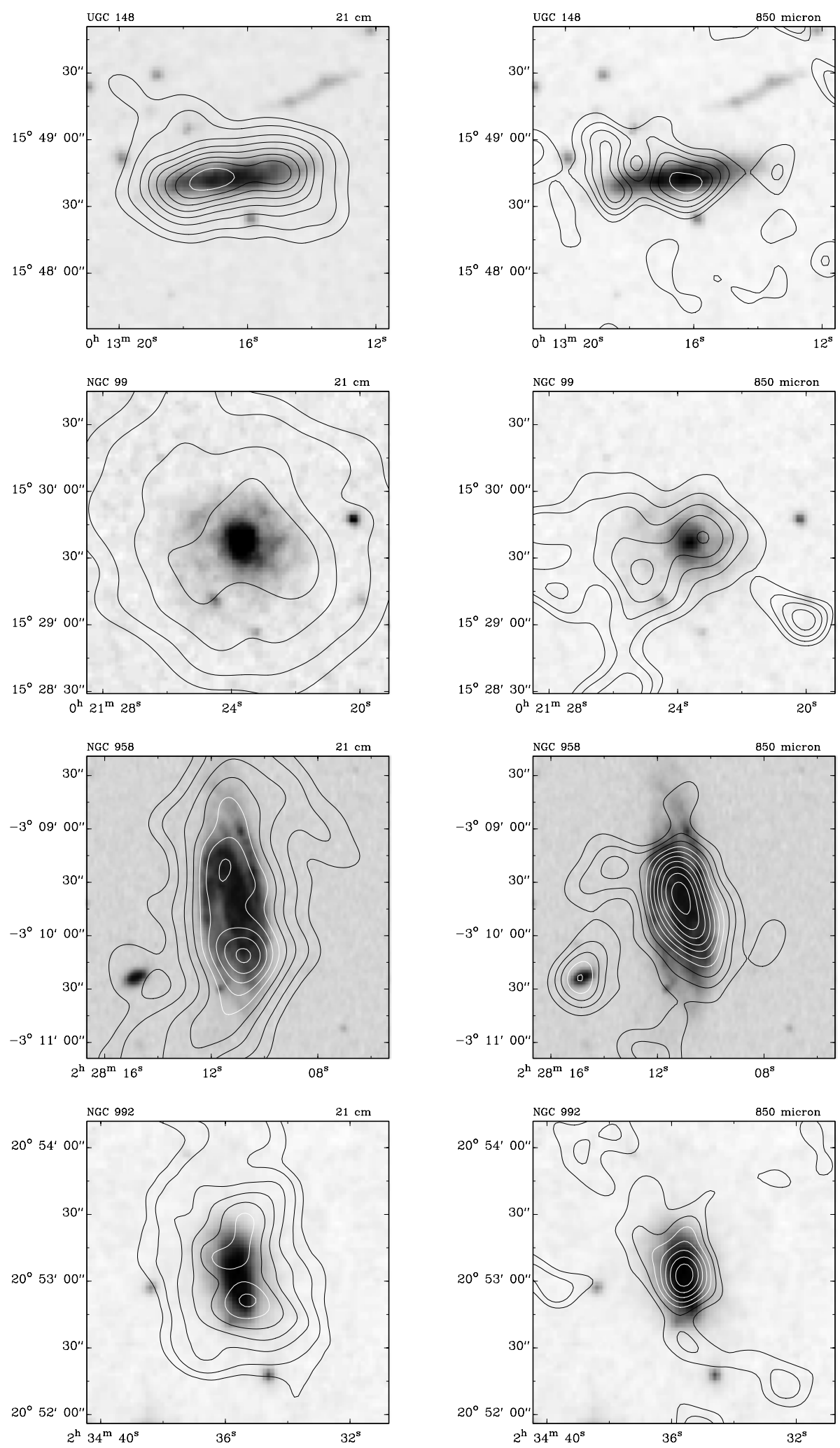

Figure 1. VLA C-array H I images, with continuum subtracted (left) and SCUBA 850- $\mu \mathrm{m}$ images (right) convolved to a resolution of 25 arcsec and overlaid on an optical $\left(R\right.$-band) Digitized Sky Survey image. The contours for the $\mathrm{H}$ I images are $\pm(0.2,0.5,1.0,1.5,2.0 \ldots) \times 10^{21}$ atom $\mathrm{cm}^{-2}$, where negative contours are dashed, and positive contours solid. The contours for the $850-\mu \mathrm{m}$ images are $10,20,30,40 \ldots \mathrm{mJy} \mathrm{beam}^{-1}$. All images are 2.3 arcmin in extent, which is the SCUBA field of view. (Larger field of view images of NGC 4793, 5020, 5962 and 7591 are shown in Fig. 2.) 

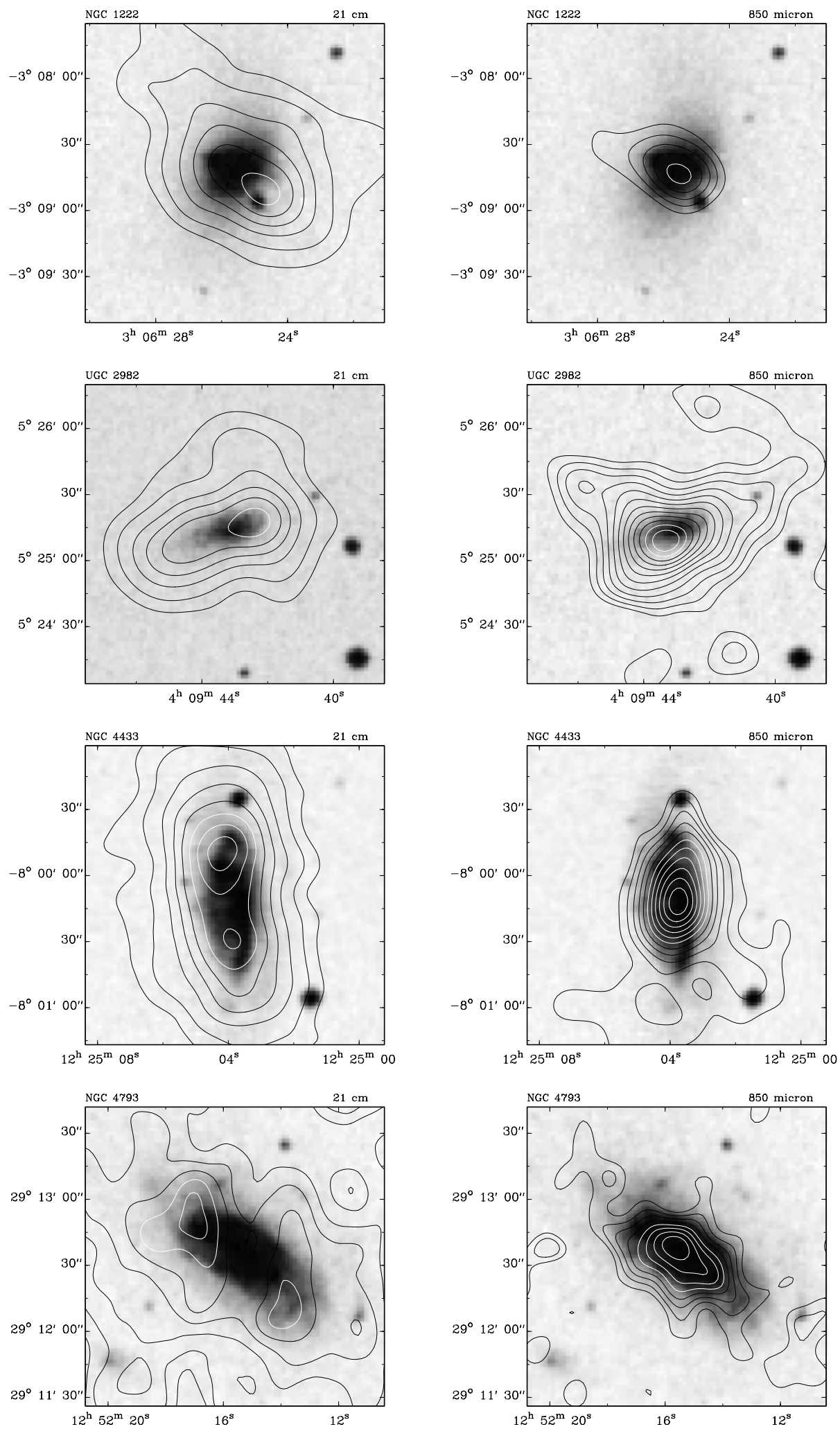

Figure 1 - continued

local H I minimum, often, but not always, at the centre of the galaxy. This anti-correlation has three possible explanations as follows.

(i) H I absorption against the radio continuum emission accompanying the $850-\mu \mathrm{m}$ flux reduces the observed line flux. (ii) The dust mass distribution is similar to the H I distribution but the dust temperature or emissivity rises sharply in the centre of the sources; due to either a starburst nucleus or active galactic nucleus.

(iii) Conversion of $\mathrm{HI}$ to $\mathrm{H}_{2}$ is greater in dusty regions. 

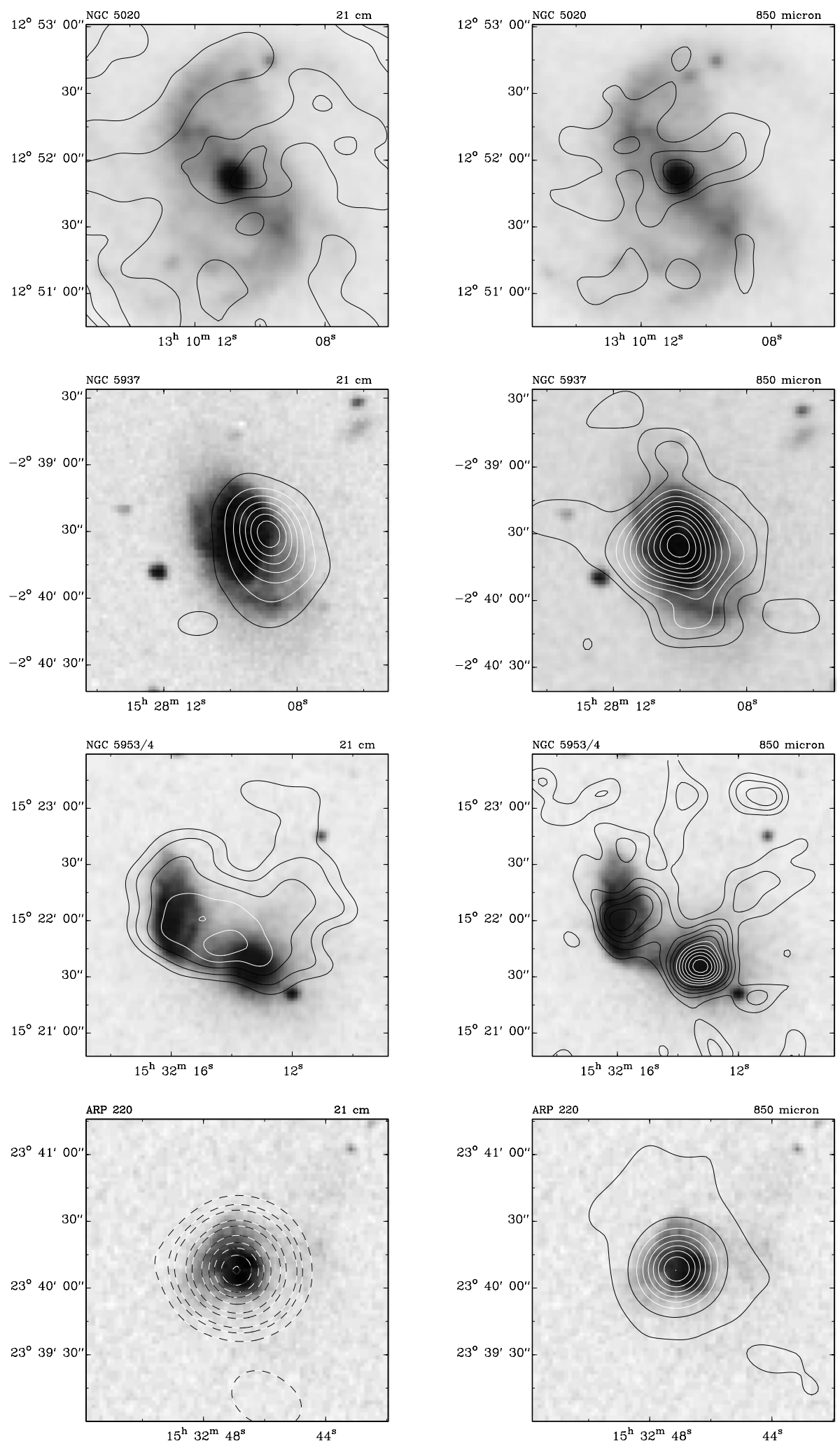

Figure 1 - continued

As discussed in Paper I, the brightness temperature of the continuum from the centre of galaxies, as observed at $1.4 \mathrm{GHz}$ with a 20 -arcsec beam, is large compared with the typical Hi emission observed. Consequently even very small optical depths will produce significant absorption towards the centres of these galaxies compared with the $\mathrm{HI}$ emission. In addition, those galaxies that show a central $\mathrm{HI}$ minimum tend to have higher inclinations than those that do not, which favours the absorption hypothesis. 

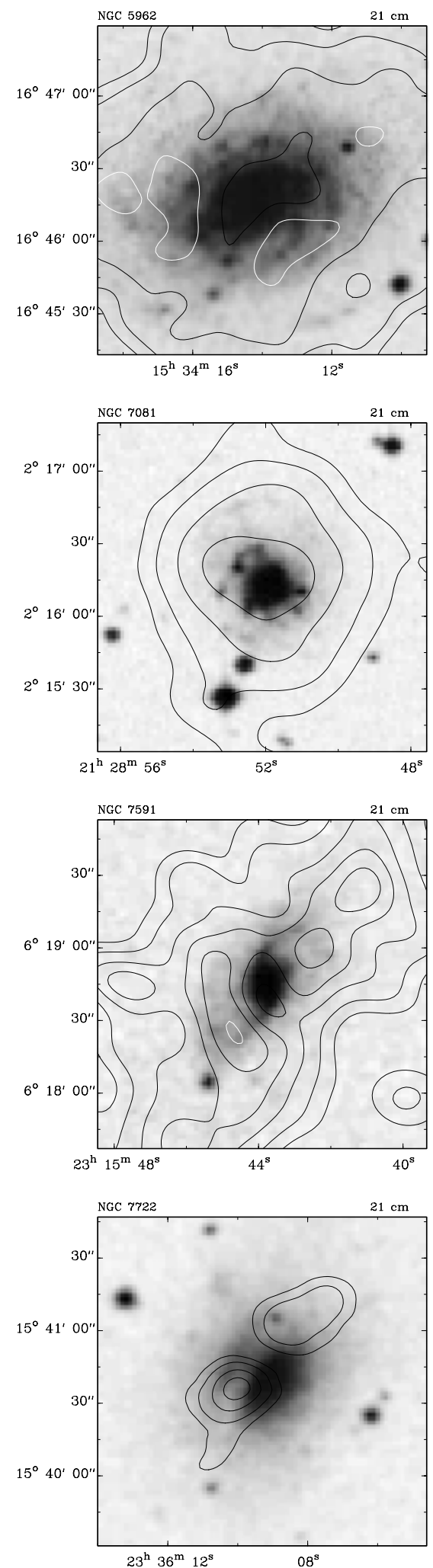
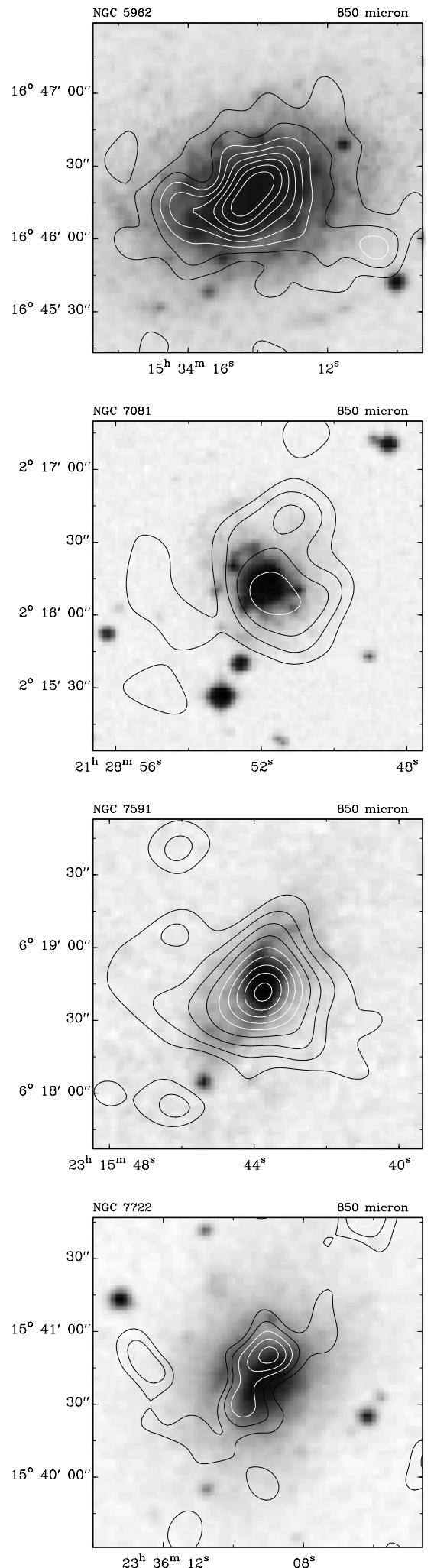

Figure 1 - continued

The second possibility requires spatially resolved data that trace the dust temperature, which we do not have. The $450-\mu \mathrm{m}$ data from the SLUGS survey are generally not of sufficient quality to trace the radial temperature profile. However, even for an AGN-dominated source such as NGC 4418, Dunne \& Eales (2001) find a dust temperature no higher than $60 \mathrm{~K}$ for the warm component. As the $850-\mu \mathrm{m}$ flux is approximately proportional to temperature, it seems unlikely that the large rise in far-infrared emission towards the centre of these 

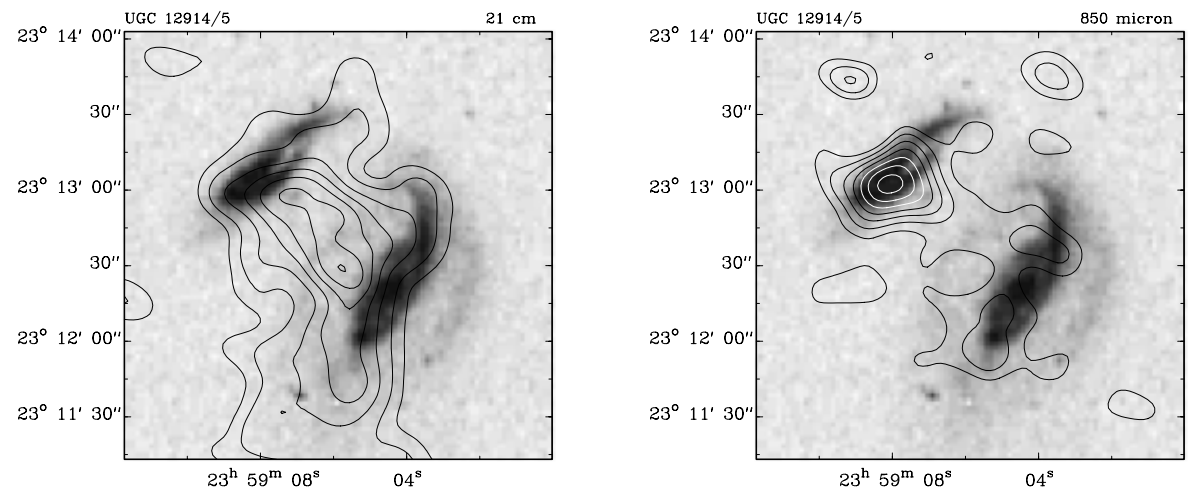

Figure 1 - continued
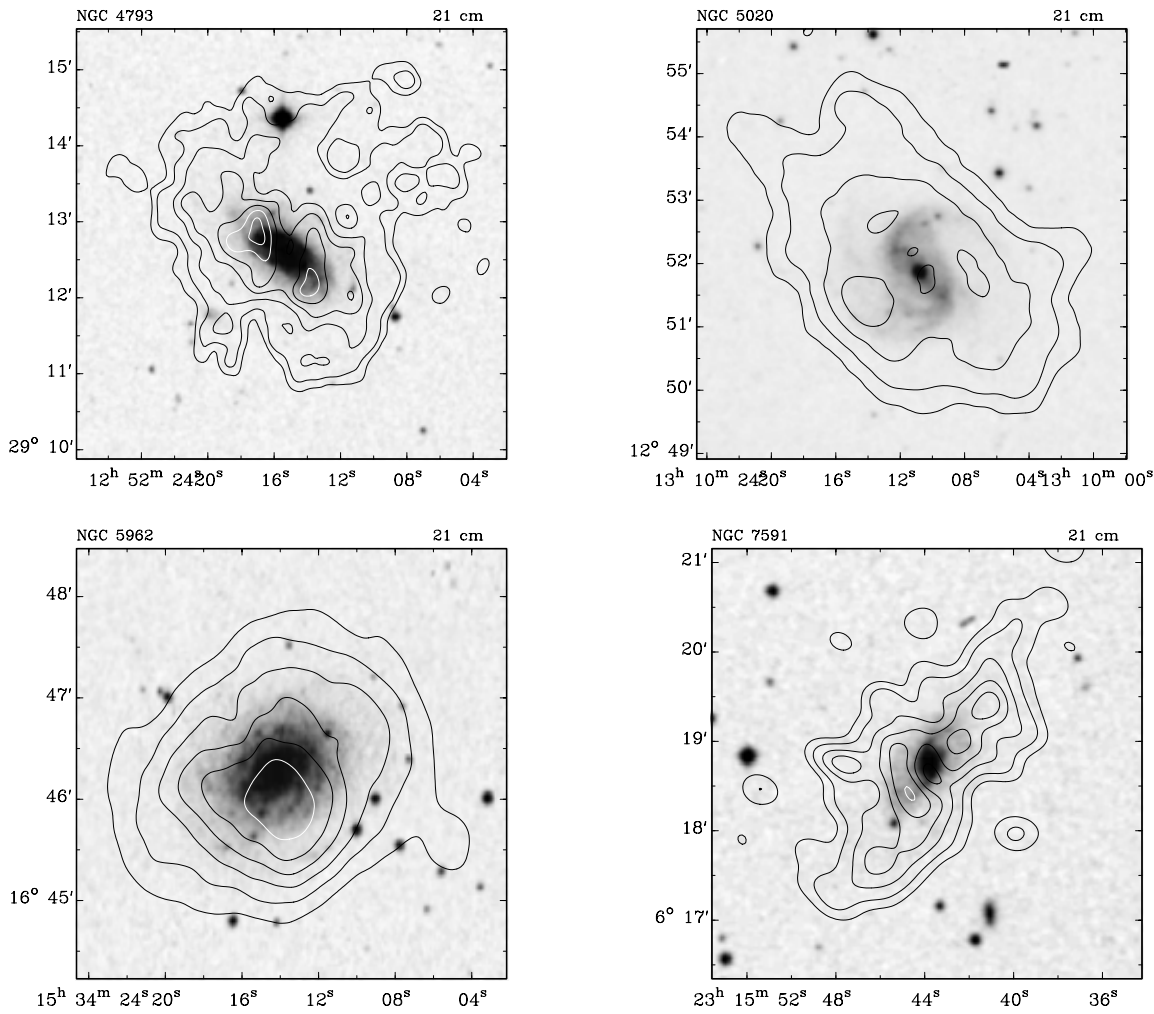

Figure 2. VLA C-array H I images of NGC 4793, 5020, 5962 and 7591, covering a wider field than shown in Fig. 1. The contours are $\pm(0.2,0.5,1.0,1.5$, $2.0 \ldots) \times 10^{21}$ atom $\mathrm{cm}^{-2}$. The NGC 4793 and 7591 images are from C-array data alone, and have a resolution of 25 arcsec. The NGC 5020 and 5962 images are from combined C- and D-array data, and have a resolution of 40 arcsec.

sources could be due entirely to a temperature increase. It is unlikely that this can explain the frequent coincidence of $\mathrm{H}$ I minima with 850- $\mu$ m maxima.

If $\mathrm{H}$ I had been processed to $\mathrm{H}_{2}$ in the centre of these sources, as might be expected for a central starburst for example, we would expect to see a central concentration of molecular gas. CO observations of the objects are needed in order to test this possibility.

As H I absorption is almost certainly important for most of the sources in this sample the importance of this effect would need to be determined before considering the possibility of processing between atomic and molecular gas components.

\subsection{Gas and dust masses}

Combining the results from this paper with those presented in Paper $\mathrm{I}$, a comparison of the overall $\mathrm{H}$, dust and $\mathrm{H}_{2}$ masses of the IRAS

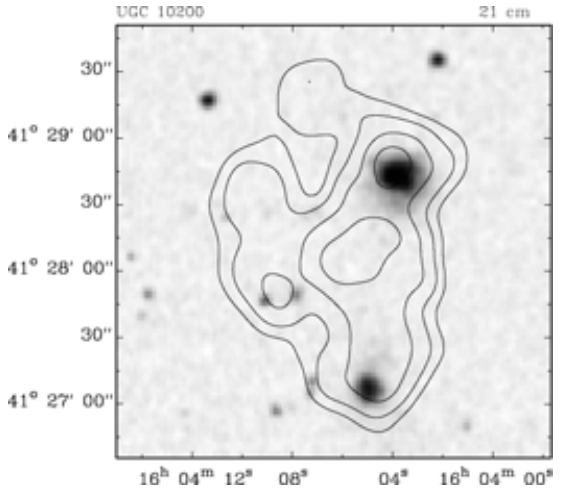

Figure 3. VLA C-array H I image of UCG 10200, which was not detected

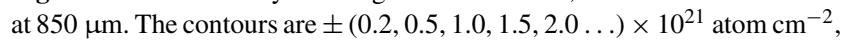
and the resolution is 25 arcsec. 

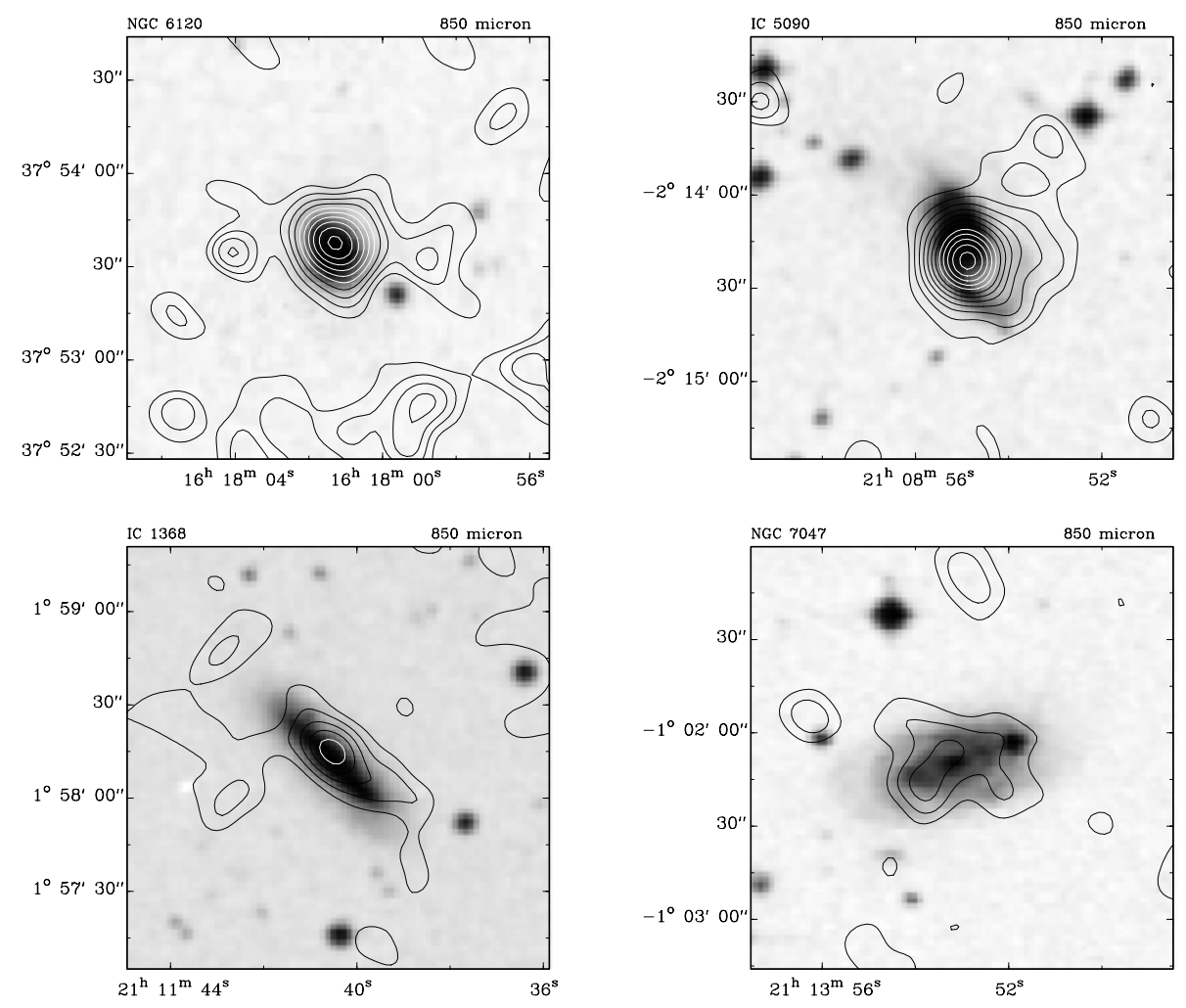

Figure 4. SCUBA $850-\mu \mathrm{m}$ images of galaxies not detected, or only weakly detected, in $\mathrm{H}$. The data have been smoothed to a resolution of 20 arcsec. The contours are at $5,10,15,20 \ldots$ mJy beam $^{-1}$.

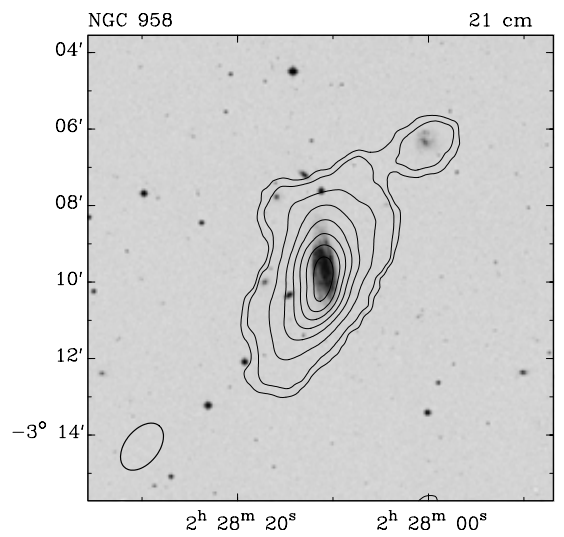

Figure 5. VLA H I image of NGC 958, from VLA D-array data. The contours are $\pm(0.5,1,3,6,9 \ldots) \times 10^{20}$ atom $\mathrm{cm}^{-2}$, and the resolution is 124 $\times 60 \operatorname{arcsec}^{2}$, as indicated by the ellipse in the bottom left corner.

bright sample galaxies can be made. Excluding galaxies in pairs, and those only detected in absorption, $\mathrm{H}$ I and dust masses are available for 20 galaxies, and the $\mathrm{H} \mathrm{I} /$ dust mass ratio averaged over two bins by morphological type (Sa to Sbc, and Sc to Sd, each with 10 galaxies) are given in Table 3 . For 14 of these galaxies $\mathrm{H}_{2}$ masses are also available, and the $\mathrm{H}_{2}$ /dust, $\mathrm{H}$ I/ $\mathrm{H}_{2}$ and gas/dust (i.e. $\mathrm{HI}$ and $\mathrm{H}_{2}$ to dust) mass ratios for these galaxies are also given in Table 3. From these results, none of the mass ratios shows any obvious dependence on morphological type.

\section{CONCLUSIONS}

We have presented further matched-resolution $\mathrm{HI}$ and $850-\mu \mathrm{m}$ images of galaxies from the SLUGS sample. Most galaxies are from the IRAS bright sample in SLUGS, but some are from an optically selected sample. Although the $850-\mu \mathrm{m}$ emission generally peaks near the centres of the galaxies, the H I images often show minima, which are probably due to absorption. For the IRAS selected galaxies, these observations, along with results previously presented, do not show any obvious trends in the $\mathrm{H} \mathrm{I} /$ dust or $\mathrm{H}_{2} /$ dust mass ratios with morphological type.

\section{ACKNOWLEDGMENTS}

The National Radio Astronomy Observatory is a facility of the $\mathrm{Na}$ tional Science Foundation operated under cooperative agreement by Associated Universities, Inc. The JCMT is operated by the Joint

Table 3. The mean properties of $M_{\mathrm{HI}} / M_{\mathrm{d}}, M_{\mathrm{H}_{2}} / M_{\mathrm{d}}, M_{\mathrm{H}_{\mathrm{I}}} / M_{\mathrm{H}_{2}}$ and $M_{\mathrm{g}} / M_{\mathrm{d}}$ as a function of morphological type.

\begin{tabular}{lcccccc}
\hline Type & Number & $\left\langle\frac{M_{\mathrm{HI}}}{M_{\mathrm{d}}}\right\rangle$ & Number with $M_{\mathrm{H}_{2}}$ & $\left\langle\frac{M_{\mathrm{H}_{2}}}{M_{\mathrm{d}}}\right\rangle$ & $\left\langle\frac{M_{\mathrm{HI}}}{M_{\mathrm{H}_{2}}}\right\rangle$ & $\left\langle\frac{M_{\mathrm{g}}}{M_{\mathrm{d}}}\right\rangle$ \\
\hline $\mathrm{Sa} / \mathrm{Sab} / \mathrm{Sb} / \mathrm{Sbc}$ & 10 & $173 \pm 45$ & 7 & $270 \pm 74$ & $2.14 \pm 0.92$ & $474 \pm 118$ \\
$\mathrm{Sc} / \mathrm{Scd} / \mathrm{Sd}$ & 10 & $197 \pm 35$ & 7 & $206 \pm 101$ & $1.19 \pm 0.35$ & $381 \pm 123$ \\
All types & 20 & $185 \pm 28$ & 14 & $238 \pm 61$ & $1.67 \pm 0.49$ & $428 \pm 83$ \\
\hline
\end{tabular}


Astronomy Centre on behalf of the UK Particle Physics Research Council, the Netherlands Organization for Scientific Research and the Canadian National Research Council. The NASA/IPAC Extragalactic Data base (NED) is operated by the Jet Propulsion Laboratory, California Institute of Technology, under contract with the National Aeronautics and Space Administration. The Digitized Palomar Sky Survey was funded by the National Geographic Society and produced by the Space Science Telescope Institute from plates taken from the Oschin Schmidt Telescope. This is operated jointly by the California Institute of Technology and the Palomar Observatory.

\section{REFERENCES}

Anantharamaiah K. R., Viallefond F., Mohan N. R., Goss W. M., Zhao J. H., 2000, ApJ, 537, 613

Casoli F., Dickey J., Kazès I., Boselli A., Gavazzi G., Jore K., 1996, A\&AS, 116, 193

Chini R., Krügel E., Lemke R., 1996, A\&AS, 118, 47

Condon J. J., Helou G., Sanders D. B., Soifer B. T., 1993, AJ, 105, 1730

Dunne L., 2000, PhD thesis, University of Wales Cardiff

Dunne L., Eales S. A., 2001, MNRAS, 327, 697

Dunne L., Eales S., Edmunds M., Ivison R., Alexander P., Clements D. L., 2000, MNRAS, 315, 115

Elfhag T., Booth R. S., Höglund B., Johansson L. E. B., Sandqvist A., 1996, A\&AS, 115, 439

Huchra J. P., Vogeley M. S., Geller M. J., 1999, ApJS, 121, 287

Kenney J. D., Young J. S., 1989, ApJ, 344, 171

Lavezzi T. E., Dickey J. M., 1998, AJ, 116, 2672

McDowell J. C. et al., 2003, ApJ, 591, 154

Maiolino R., Ruiz M., Rieke G. H., Papadopoulos P., 1997, ApJ, 485, 552
Sanders D. B., Mirabel I. F., 1985, ApJ, 298, L31

Sanders D. B., Scoville N. Z., Soifer B. T., 1991, ApJ, 370, 158

Sofue Y., Wakamatsu K., Taniguchi Y., Nakai N., 1993, PASJ, 45, 43

Soifer B. T., Neugebauer G., Matthews K., Becklin E. E., Ressler M., Werner M. W., Weinberger A. J., Egami E., 1999, ApJ, 513, 207

Theureau G., Bottinelli L., Coudreau-Durand N., Gouguenheim L., Hallet N., Loulergue M., Paturel G., Teerikorpi P., 1998, A\&AS, 130, 333

Thomas H. C., Dunne L., Clemens M. S., Alexander P., Eales S., Green D. A., 2002, MNRAS, 329, 747 (Paper I)

Thomas H. C., Alexander P., Clemens M. S., Green D. A., Dunne L., Eales S., 2004, MNRAS, submitted (Paper III)

Tinney C. G., Scoville N. Z., Sanders D. B., Soifer B. T., 1990, ApJ, 362, 473

Wang Z., Kenney J. D., Ishizuki S., 1992, AJ, 104, 2097

Wiedner M. C., Wilson C. D., Harrison A., Hills R. E., Lay O. P., Carlstrom J. E., 2002, ApJ, 581, 229

Young J. S. et al., 1995, ApJS, 98, 219

Zhu M., Seaquist E. R., Davoust E., Frayer D. T., Bushouse H. A., 1999, AJ, 118,145

\section{APPENDIX A：D-ARRAY OBSERVATIONS}

As noted above, a small sample of 13 of the larger (greater than 2.5 arcmin) SLUGS galaxies were observed with the VLA in Darray. These data were analysed in a similar way to the C-array data (see Section 2). Three of the galaxies observed (NGC 958, 5020 and 5962) were also observed in C-array, and the results for these galaxies are given above. Of the other galaxies observed, one (UGC 2369) was not detected in the D-array observations. The integrated $\mathrm{H}$ I images for the other nine galaxies are presented in Fig. A1. 

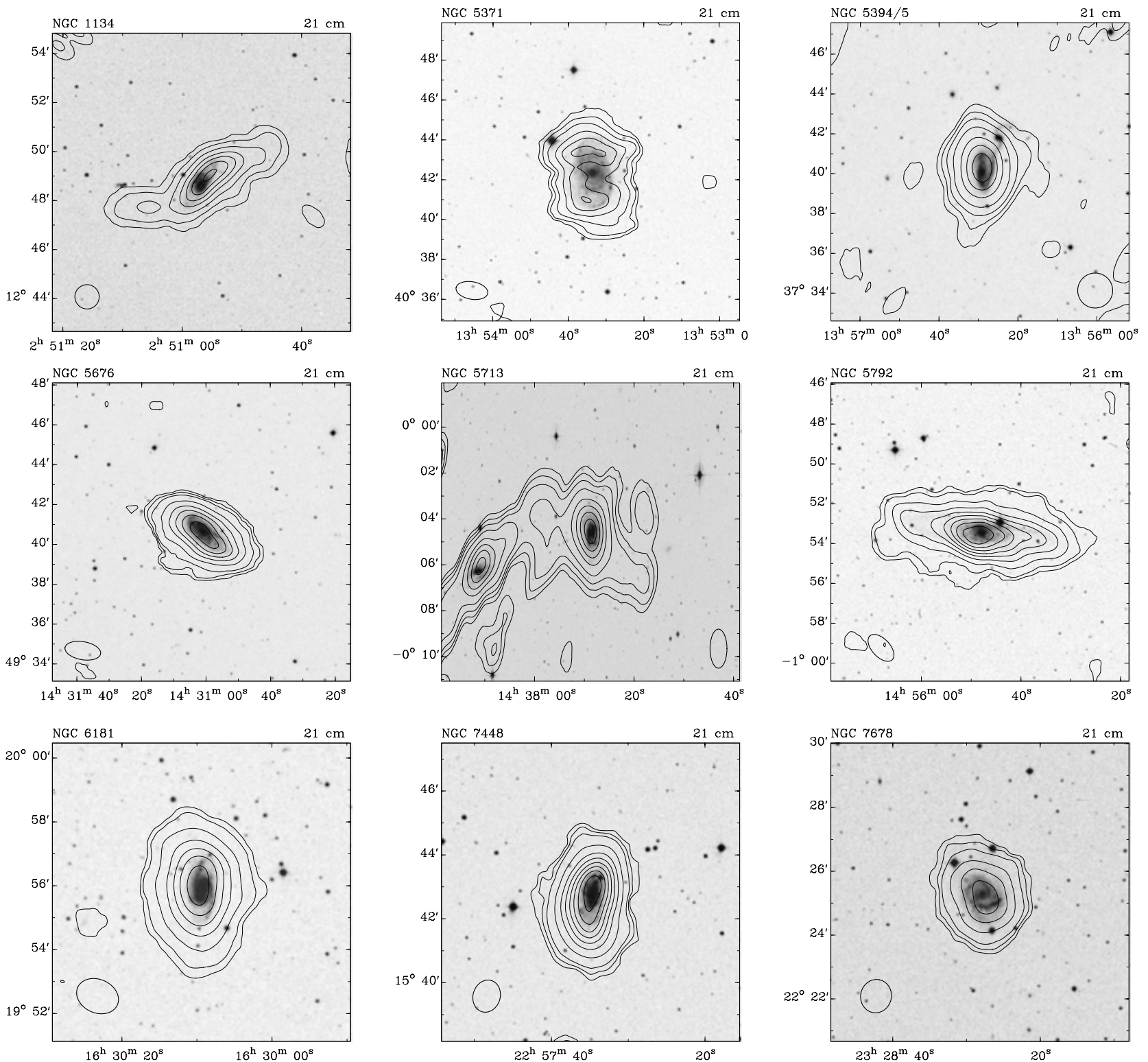

Figure A1. VLA H I images of galaxies observed in D-array. The contours are $\pm(0.5,1,3,6,9 \ldots) \times 10^{20}$ atom $\mathrm{cm}^{-2}$, and the resolution of each image is indicated by the ellipse in the bottom corner.

This paper has been typeset from a $\mathrm{T}_{\mathrm{E}} \mathrm{X} / \mathrm{L} \mathrm{A} \mathrm{T} \mathrm{X}$ file prepared by the author. 